\title{
Sylvester versus Gundelfinger ${ }^{\star}$
}

\author{
Andries E. BROUWER ${ }^{\dagger}$ and Mihaela POPOVICIU ${ }^{\ddagger}$ \\ $\dagger$ Department of Mathematics and Computer Science, Technische Universiteit Eindhoven, \\ P.O. Box 513, 5600 MB Eindhoven, The Netherlands \\ E-mail: aeb@cwi.nl \\ URL: http://www.win.tue.nl/ aeb/ \\ $\ddagger$ Mathematisches Institut, Universität Basel, Rheinsprung 21, CH-4051 Basel, Switzerland \\ E-mail: mihaela.popoviciu@unibas.ch
}

Received July 18, 2012, in final form October 12, 2012; Published online October 19, 2012

http://dx.doi.org/10.3842/SIGMA.2012.075

\begin{abstract}
Let $V_{n}$ be the $\mathrm{SL}_{2}$-module of binary forms of degree $n$ and let $V=V_{1} \oplus V_{3} \oplus V_{4}$. We show that the minimum number of generators of the algebra $R=\mathbb{C}[V]^{\mathrm{SL}_{2}}$ of polynomial functions on $V$ invariant under the action of $\mathrm{SL}_{2}$ equals 63 . This settles a 143-year old question.
\end{abstract}

Key words: invariants; covariants; binary forms

2010 Mathematics Subject Classification: 13A15; 68W30

\section{Introduction}

In 1868 Gordan [4] proved that the algebra of invariants of binary forms of given degree is finitely generated. This came as a surprise to Cayley and Sylvester, who had believed that the quintic and septimic had covariant resp. invariant rings that were not finitely generated.

The number of invariants is first infinite in the case of a quantic of the seventh order, or septic; the number of covariants is first infinite in the case of a quantic of the fifth order, or quintic. (Cayley [2])

However, finding a minimal set of generators for these algebras is even today an open problem in all but a few small cases. In the case of $V_{4} \oplus V_{4}$, Gordan found a generating set of size 30, and Sylvester [17] showed that two of these generators are superfluous. He added

J'ajouterai seulement que cette preuve éclatante de l'insuffisance de la méthode de M. Gordan et de son école, pour séparer les formes véritablement élémentaires des formes superflues qui s'y rattachent (insuffisance reconnue par M. Gordan lui-même de la manière la plus loyale dans son discours inaugural prononcé à Erlangen), n'ôte rien à la valeur immense du service qu'il a rendu à l'Algèbre, en ayant le premier démontré l'existence d'une limite au nombre de ces formes.

This note focuses on the covariants of $V_{3} \oplus V_{4}$, a case which illustrates the controversy between the German and English schools in the 19th century. The German school, following Clebsch and Gordan, was able to construct a system of generators for the algebra of invariants of binary forms, with no guarantee that the system was minimal. The English school, following Cayley and Sylvester, aimed to determine the number of independent generators. Sylvester used in his computations his 'fundamental postulate' (not defined here), which turned out to hold only in small cases. Counterexamples were given by Hammond [7] and Morley [10].

\footnotetext{
${ }^{\star}$ This paper is a contribution to the Special Issue "Symmetries of Differential Equations: Frames, Invariants and Applications". The full collection is available at http://www.emis.de/journals/SIGMA/SDE2012.html
} 
In 1869 Gundelfinger, a student of Clebsch, wrote a thesis [6] where he constructed generators for the covariants of $V_{3} \oplus V_{4}$ 'in ordinary symbolic notation', after Clebsch had given him this system as computed by Gordan in his 'obscure' notation (cf. [5, pp. 270-272]). He found 20 generators for the invariants and 64 for the covariants.

Sylvester used the Poincaré series together with his fundamental postulate to show that there could be only 61 independent generators for the covariants of $V_{3} \oplus V_{4}$, and wrote a series of papers $[15,18,19,20]$ showing the superiority of the English methods over the German.

In the first paper Sylvester uses his method (which he calls 'tamisage') to derive the numbers of generators of given degrees in the coefficients of $V_{4}$ and those of $V_{3}$, and given order in the variables $x, y$. The following table is taken from [20]:

Table of Groundforms.*

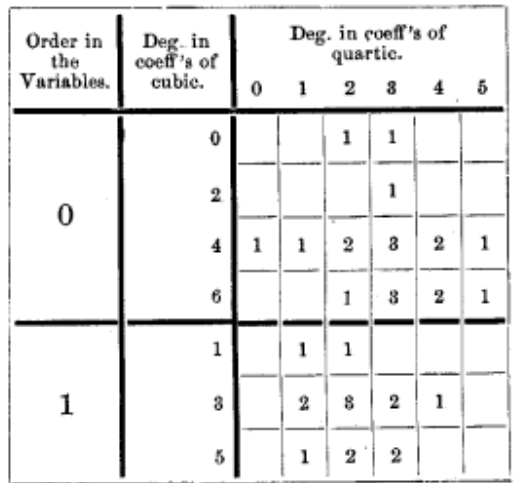

\begin{tabular}{|c|c|c|c|c|c|}
\hline \multirow{2}{*}{$\begin{array}{c}\text { Order in } \\
\text { the } \\
\text { Varisbles }\end{array}$} & \multirow{2}{*}{$\begin{array}{l}\text { Deg, in } \\
\text { coeff's of } \\
\text { eubic. }\end{array}$} & \multicolumn{4}{|c|}{$\begin{array}{l}\text { Deg. in coeff's of } \\
\text { quartic. }\end{array}$} \\
\hline & & 0 & 1 & 2 & 3 \\
\hline \multirow{2}{*}{2} & 2 & 1 & 2 & 2 & 1 \\
\hline & 4 & & 2 & 2 & \\
\hline \multirow{2}{*}{3} & 1 & 1 & 1 & 1 & 1 \\
\hline & 8 & 1 & 1 & 1 & 1 \\
\hline \multirow{2}{*}{4} & 0 & & 1 & 1 & \\
\hline & 2 & & 1 & 1 & 1 \\
\hline 5 & 1 & & 1 & 1 & \\
\hline 6 & 0 & & & & 1 \\
\hline
\end{tabular}

\footnotetext{
* The form of ord. 1, deg. 5, 4, and the two forms of ord. 2, deg. 4, 3, given by Gundelfinger, do not appenr in this table, and it has been proved by the author that no fundamental forms of elther of these types exist.
}

In the second paper he observes that it follows from the Poincaré series that there are 8 linearly independent covariants of order 2 and multidegree $(4,3)$. Next, he constructs 8 reducible such covariants (products of covariants of lower degree) and argues that these are linearly independent. However, the forms are dependent and only seven are independent. He finishes with the announcement

Dans une prochaine Communication j'entreprendrai l'examen de la seule forme qui reste à discuter, c'est-à-dire le covariant linéaire des degrés 4, 5 dans les coefficients, qui se trouve dans la Table de $\mathbf{M}$. Gundelfinger, mais en dehors de la mienne. On sait déjà que le nombre des formes irréductibles pour le système en question est ou 61 ou 62 . Il me semble peu douteux que c'est le premier de ces nombres qui sortira victorieux de la discussion du type 4.5.1.

In the third paper he observes that it follows from the Poincaré series that there are 12 linearly independent covariants of order 1 and multidegree $(5,4)$. Next, he constructs 12 reducible such covariants and argues that these are linearly independent. However, the forms are dependent and only eleven are independent. He concludes

Done il n'y a nul covariant irréductible du type 4.5.1, et conséquemment le montant des grundformen pour le système cubo-biquadratique binaire est 61 , comme j'ai trouvé, et non pas 64 comme M. Gundelfinger avait pensé.

Je conclus par l'observation importante que ms méthode serait parfaitement démontrée de priori si l'on pouvait démontrer le théorème suivant: 
(false theorem omitted)

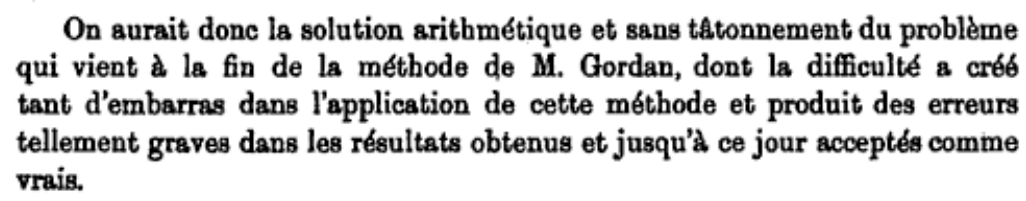

Here we show that the actual minimal number of generators for the covariants of $V_{3} \oplus V_{4}$ is 63 . Our results coincide with those of Sylvester and Gundelfinger, with two exceptions: we show that one needs one generating covariant of order 1 and multidegree $(5,4)$, and (only) one generating covariant of order 2 and multidegree $(4,3)$.

For completeness we give the corrected version of Sylvester's table. The two corrected entries are underlined.

\begin{tabular}{|c|c|c|c|c|c|c|c|c|c|c|c|c|c|}
\hline \multirow{3}{*}{ Order } & \multirow{3}{*}{$\begin{array}{l}\text { Deg. in } \\
\text { coeff's of } \\
\text { cubic. }\end{array}$} & \multirow{2}{*}{\multicolumn{6}{|c|}{$\begin{array}{l}\text { Deg. in coeff's } \\
\text { of quartic. }\end{array}$}} & \multirow[t]{2}{*}{ Order } & \multirow{2}{*}{$\begin{array}{l}\text { Deg. in } \\
\text { coeff's of } \\
\text { cubic. }\end{array}$} & \multicolumn{4}{|c|}{$\begin{array}{l}\text { Deg. in coeff's } \\
\text { of quartic. }\end{array}$} \\
\hline & & & & & & & & & & & 1 & 2 & 3 \\
\hline & & 0 & 1 & 2 & 3 & 4 & 5 & \multirow[b]{2}{*}{2} & 2 & \multirow[t]{2}{*}{$\overline{1}$} & \multirow{2}{*}{\multicolumn{3}{|c|}{$\begin{array}{l}1 \\
1\end{array}$}} \\
\hline \multirow{4}{*}{0} & 0 & & & 1 & 1 & & & & 4 & & & & \\
\hline & 2 & & & & 1 & & & \multirow{2}{*}{3} & 1 & 1 & 1 & 1 & 1 \\
\hline & 4 & 1 & 1 & 2 & 3 & 2 & 1 & & 3 & 1 & 1 & 1 & 1 \\
\hline & & & & 1 & 3 & 2 & 1 & \multirow[b]{2}{*}{4} & $\overline{0}$ & & 1 & $\overline{1}$ & \multirow{3}{*}{1} \\
\hline \multirow[b]{2}{*}{1} & 1 & & 1 & 1 & & & & & 2 & & 1 & 1 & \\
\hline & $\begin{array}{l}3 \\
5\end{array}$ & & $\begin{array}{l}2 \\
1\end{array}$ & $\begin{array}{l}3 \\
2\end{array}$ & $\begin{array}{l}2 \\
2\end{array}$ & $\begin{array}{l}1 \\
1\end{array}$ & & 5 & 1 & & 1 & 1 & \\
\hline & & & & & & & & 6 & 0 & & & & 1 \\
\hline
\end{tabular}

\subsection{Invariants and covariants}

Let $V$ be a finite-dimensional vector space over a field $k$, with basis $e_{1}, \ldots, e_{m}$. Let $x_{i}$ be the coordinate function defined by $x_{i}\left(\sum \xi_{h} e_{h}\right)=\xi_{i}$. The algebra $k[V]$ of polynomial functions on $V$ is by definition the algebra generated by the $x_{i}$. (It does not depend on the choice of basis $e_{1}, \ldots, e_{m}$.) Let $G$ be a group of linear transformations of $V$. It acts on $k[V]$ via the action $(g \cdot f)(v)=f\left(g^{-1} v\right)$. Invariant theory studies $k[V]^{G}$, the algebra of $G$-invariant polynomial functions on $V$, i.e., the $f \in k[V]$ such that $g \cdot f=f$ for all $g \in G$.

A covariant of order $m$ and degree $d$ of $V$ is a $G$-equivariant homogeneous polynomial map $\phi: V \rightarrow V_{m}$ of degree $d$. In other words, $\phi(g \cdot v)=g \cdot \phi(v)$, for all $g \in G$, and $\phi(t v)=t^{d} \phi(v)$, for all $t \in k$. In particular, the covariants of $V$ of order 0 are the invariants of $V$.

Below we shall take $k=\mathbb{C}, G=\mathrm{SL}_{2}(k)$, and $V=V_{n_{1}} \oplus \cdots \oplus V_{n_{p}}$, where $V_{n}$ is the vector space (of dimension $n+1$ ) consisting of 0 and the binary forms of degree $n$, that is, of the homogeneous polynomials of degree $n$

$$
v(x, y)=a_{0} x^{n}+\left(\begin{array}{c}
n \\
1
\end{array}\right) a_{1} x^{n-1} y+\cdots+\left(\begin{array}{c}
n \\
n-1
\end{array}\right) a_{n-1} x y^{n-1}+a_{n} y^{n},
$$

in two variables. This $V_{n}$ is the $n$-th graded part of $k[W]$, where $W$ is a 2-dimensional vector space over $\mathbb{C}$ with natural action of $\mathrm{SL}_{2}$, hence has a natural action of $\mathrm{SL}_{2}$.

The main way to construct covariants is via transvectants (Überschiebungen). These are derived from the Clebsch-Gordan decomposition of the $\mathrm{SL}_{2}$-module $V_{m} \otimes V_{n}$, with $m \geq n$ :

$$
V_{m} \otimes V_{n} \simeq V_{m+n} \oplus V_{m+n-2} \oplus \cdots \oplus V_{m-n} .
$$

This decomposition defines for each $p, 0 \leq p \leq n$, an $\mathrm{SL}_{2}$-equivariant linear map $V_{m} \otimes V_{n} \rightarrow$ $V_{m+n-2 p}$, denoted $(g, h) \mapsto(g, h)_{p}$, and called the $p$-th transvectant. It is given explicitly by the 
following formula:

$$
(g, h) \mapsto(g, h)_{p}:=\frac{(m-p) !(n-p) !}{m ! n !} \sum_{i=0}^{p}(-1)^{i}\left(\begin{array}{c}
p \\
i
\end{array}\right) \frac{\partial^{p} g}{\partial x^{p-i} \partial y^{i}} \frac{\partial^{p} h}{\partial x^{i} \partial y^{p-i}}
$$

(see [11, Chapter 5]).

The covariants of $V$ can be identified with the invariants of $V_{1} \oplus V$ : we have $V_{1} \oplus V \simeq V_{1}^{*} \oplus V$ as $\mathrm{SL}_{2}$-representations and the set of covariants of $V$ is isomorphic to $k\left[V_{1}^{*} \oplus V\right]^{\mathrm{SL}_{2}}$ (see [12, Chapter 15]). Each covariant $\phi$ of $V$ of order $m$ corresponds to the invariant of $V_{1} \oplus V$ defined by the transvectant $\left(\phi(v), l^{m}\right)_{m}$, where $l \in V_{1}$.

\section{The generators of the invariants of $V_{1} \oplus V_{3} \oplus V_{4}$}

We identify the covariants of $V_{3} \oplus V_{4}$ with the invariants of $V_{1} \oplus V_{3} \oplus V_{4}$ and show that a minimal set of generators for the algebra of invariants of this module has size 63 .

Doing this type of work requires finding dependencies. Gundelfinger did not try to do this exhaustively, but following Gordan he only noted the obvious ones. Sylvester tried, and made some mistakes, no doubt because he already knew what answer he wanted. For us this is relatively easy - a modern computer has no problems computing the rank of a 40000 by 600000 matrix (which is what is needed in the most straightforward approach).

We had a different problem: up to which degree should we compute covariants or invariants? Gundelfinger 'just' followed Gordan's algorithm, but as far as we know that has not been implemented yet.

The secret knowledge known today but not in the 19th century, is that the ring $R$ of invariants of $V_{1} \oplus V_{3} \oplus V_{4}$ (or any such ring) is Cohen-Macaulay (see [9]). It has a homogeneous system of parameters (hsop) $j_{1}, \ldots, j_{r}$, algebraically independent, and finitely many further generators $i_{1}, \ldots, i_{s}$, such that every invariant can be uniquely written as a linear combination of products $i_{m} j_{m_{1}} \cdots j_{m_{h}}$. It follows that the Poincaré series $P(t)=\sum d_{i} t^{i}$, where $d_{i}$ is the dimension of the degree $i$ part of $R$, is of the form

$$
P(t)=\frac{t^{a_{1}}+\cdots+t^{a_{s}}}{\left(1-t^{b_{1}}\right) \cdots\left(1-t^{b_{r}}\right)},
$$

where the $a_{h}$ and $b_{h}$ are the degrees of the $i_{h}$ and $j_{h}$.

For the module $V=V_{n_{1}} \oplus \cdots \oplus V_{n_{p}}$, with $\sum_{i} n_{i} \geq 3$, one has $P\left(t^{-1}\right)=(-1)^{d-3} t^{d} P(t)$ where $d=\sum_{i}\left(n_{i}+1\right)$ (by [14, Corollary 2] for $p=1$, and by [1, Theorem 2] in general), so that $\max _{h} a_{h}-\sum_{j} b_{j}=-\sum_{i}\left(n_{i}+1\right)$. Therefore, in order to find $\max _{h}\left\{a_{h}, b_{h}\right\}$ it suffices to find the $b_{h}$.

The power series $P(t)=\sum d_{i} t^{i}$ is known from Cayley [2] and Sylvester [16] (cf. [13, 3.3.4]). In the present case,

$$
\begin{aligned}
P(t)= & 1+t^{2}+2 t^{3}+5 t^{4}+10 t^{5}+18 t^{6}+31 t^{7}+55 t^{8}+92 t^{9}+144 t^{10}+223 t^{11}+341 t^{12} \\
& +499 t^{13}+725 t^{14}+1031 t^{15}+1436 t^{16}+1978 t^{17}+2685 t^{18}+3592 t^{19}+4761 t^{20} \\
& +6235 t^{21}+8078 t^{22}+10379 t^{23}+13226 t^{24}+16698 t^{25}+20937 t^{26}+26069 t^{27} \\
& +32230 t^{28}+39614 t^{29}+48401 t^{30}+\cdots \\
= & \frac{a(t)}{\left(1-t^{3}\right)\left(1-t^{4}\right)^{2}\left(1-t^{5}\right)^{2}\left(1-t^{6}\right)^{2}\left(1-t^{7}\right)},
\end{aligned}
$$


where

$$
\begin{aligned}
a(t)= & 1+t^{2}+t^{3}+3 t^{4}+7 t^{5}+12 t^{6}+21 t^{7}+32 t^{8}+47 t^{9}+58 t^{10}+72 t^{11}+83 t^{12}+89 t^{13} \\
& +94 t^{14}+94 t^{15}+89 t^{16}+83 t^{17}+72 t^{18}+58 t^{19}+47 t^{20}+32 t^{21}+21 t^{22}+12 t^{23} \\
& +7 t^{24}+3 t^{25}+t^{26}+t^{27}+t^{29}
\end{aligned}
$$

and it follows that computing invariants up to degree 29 suffices if we know that there is a hsop with degrees $3,4,4,5,5,6,6,7$.

\subsection{Finding a hsop}

Hilbert introduced in the 19th century the notion of nullcone. If $V$ is an $\mathrm{SL}_{2}$-module, then the nullcone $\mathcal{N}(V)$ of $V$ is the set of elements of $V$ on which all invariants of $V$ of positive degree vanish. The elements of $\mathcal{N}(V)$ are called nullforms. One can show that a binary form $f \in V_{n}$ is a nullform if and only if $f$ has a root of multiplicity $>\frac{n}{2}$ (this is a consequence of the HilbertMumford criterion, see $[3, \S 2.4 .1])$. Similarly, if we have $p$ binary forms $f_{1}, \ldots, f_{p}$ of degrees $n_{1}, \ldots, n_{p}$, then $\left(f_{1}, \ldots, f_{p}\right) \in \mathcal{N}\left(V_{n_{1}} \oplus \cdots \oplus V_{n_{p}}\right)$ if and only if $f_{1}, \ldots, f_{p}$ have a common root that has multiplicity $>\frac{n_{i}}{2}$ in $f_{i}$, for all $i=1, \ldots, p$. In our particular case, if $(l, c, q) \in V_{1} \oplus V_{3} \oplus V_{4}$, then $(l, c, q) \in \mathcal{N}\left(V_{1} \oplus V_{3} \oplus V_{4}\right)$ if and only if $l^{2} \mid c$ and $l^{3} \mid q$.

Let $\mathcal{V}(J)$ stand for the vanishing locus of $J$. The following result, due to Hilbert, gives a characterisation of homogeneous systems of parameters of $k\left[V_{n_{1}} \oplus \cdots \oplus V_{n_{p}}\right]^{\mathrm{SL}_{2}}$ as sets that define the nullcone of $\mathcal{N}\left(V_{n_{1}} \oplus \cdots \oplus V_{n_{p}}\right)$ :

Proposition 1 (Hilbert [8]). Let $V=V_{n_{1}} \oplus \cdots \oplus V_{n_{p}}$, and $R=k[V]^{\mathrm{SL}_{2}}$, and $m=n_{1}+\cdots+$ $n_{p}+p-3>0$. A set $\left\{j_{1}, \ldots, j_{m}\right\}$ of homogeneous elements of $R$ is a system of parameters of $R$ if and only if $\mathcal{V}\left(j_{1}, \ldots, j_{m}\right)=\mathcal{N}(V)$.

Let our binary forms $l \in V_{1}, c \in V_{3}, q \in V_{4}$ be

$$
\begin{aligned}
& l=c_{0} x+c_{1} y, \\
& c=a_{0} x^{3}+3 a_{1} x^{2} y+3 a_{2} x y^{2}+a_{3} y^{3}, \\
& q=b_{0} x^{4}+4 b_{1} x^{3} y+6 b_{2} x^{2} y^{2}+4 b_{3} x y^{3}+b_{4} y^{4},
\end{aligned}
$$

and consider the following invariants:

$$
\begin{array}{ll}
k_{2}=(q, q)_{4}, & k_{3}=\left((q, q)_{2}, q\right)_{4}, \\
k_{4,1}=\left((c, c)_{2},(c, c)_{2}\right)_{2}, & k_{4,2}=(l c, l c)_{4}, \\
k_{4,3}=\left(c, l^{3}\right)_{3}, & k_{5,1}=\left(\left(q,(q, q)_{2}\right)_{1}, c^{2}\right)_{6}, \\
k_{5,2}=\left(\left(q, c^{2}\right)_{2}, c^{2}\right)_{6}, & k_{5,3}=\left(q, l^{4}\right)_{4}, \\
k_{6,1}=\left(\left[(c, c)_{2}\right]^{2},(q, q)_{2}\right)_{4}, & k_{6,2}=\left((l c, l c)_{2}, l c\right)_{4}, \\
k_{6,3}=\left((q, q)_{2}, l^{4}\right)_{4}, & k_{7}=\left(c^{4}, q^{3}\right)_{12} .
\end{array}
$$

We prove the following

Proposition 2. With the notations above, the invariants

$$
\begin{array}{llll}
j_{1}=k_{3}, & j_{2}=k_{4,1}+k_{2}^{2}, & j_{3}=k_{4,2}+k_{4,3}-k_{2}^{2}, & j_{4}=k_{5,1}+k_{5,2}, \\
j_{5}=k_{5,3}, & j_{6}=k_{6,1}+k_{6,2}, & j_{7}=k_{6,3}, & j_{8}=k_{7},
\end{array}
$$

(of degrees $3,4,4,5,5,6,6,7$, respectively) form a system of parameters of $k\left[V_{1} \oplus V_{3} \oplus V_{4}\right]^{\mathrm{SL}_{2}}$. 
Proof. We show that $\mathcal{V}\left(j_{1}, \ldots, j_{8}\right)=\mathcal{N}\left(V_{1} \oplus V_{3} \oplus V_{4}\right)$. Consider three cases.

Case 1: $q=0$. In this case, the vanishing of $j_{1}, \ldots, j_{8}$ reduces to $k_{4,1}=k_{4,2}+k_{4,3}=k_{6,2}=0$, which implies that $(l, c) \in \mathcal{N}\left(V_{1} \oplus V_{3}\right)$. Indeed, if $k_{4,1}=0$, then $c$ is a nullform, and, without loss of generality, we may suppose that $x^{2} \mid c$, i.e. $a_{2}=a_{3}=0$. But then $k_{6,2} \sim a_{1}^{3} c_{1}^{3}$ (we use ' $\sim$ ' for equality up to a nonzero constant). If $c_{1}=0$, then $(l, c) \in \mathcal{N}\left(V_{1} \oplus V_{3}\right)$. If $c_{1} \neq 0$, then $a_{1}=0$ and $k_{4,2}+k_{4,3} \sim a_{0} c_{1}^{3}$. Hence $a_{0}=0$, so that $c=0$ and $(l, c) \in \mathcal{N}\left(V_{1} \oplus V_{3}\right)$.

Case 2: $l=0$. In this case, the vanishing of $j_{1}, \ldots, j_{8}$ reduces to $k_{2}=k_{3}=k_{4,1}=k_{5,1}+k_{5,2}=$ $k_{6,1}=k_{7}=0$, which implies that $(c, q) \in \mathcal{N}\left(V_{3} \oplus V_{4}\right)$. Indeed, the vanishing of $k_{2}, k_{3}, k_{4,1}$ implies that $c$ and $q$ are nullforms. If $c$ or $q$ vanish identically, then the statement is clear. Otherwise, if the double zero of $c$ and the triple zero of $q$ do not coincide, we may suppose, without loss of generality, that $x^{2} \mid c$ and $y^{3} \mid q$, i.e. $a_{2}=a_{3}=b_{0}=b_{1}=b_{2}=0$. Then $k_{6,1} \sim a_{1}^{4} b_{3}^{2}$. If $a_{1}=0$, then $k_{5,1}+k_{5,2} \sim a_{0}^{2} b_{3}^{3}$, and $k_{7} \sim a_{0}^{4} b_{4}^{3}$, which contradicts the assumption $c, q \neq 0$. If $b_{3}=0$, then $k_{5,1}+k_{5,2} \sim a_{1}^{4} b_{4}$ and $k_{7} \sim a_{0}^{4} b_{4}^{3}$, which again contradicts the assumption $c, q \neq 0$.

Case 3: $q, l \neq 0$. In this case, $j_{5}=0$ implies that $q$ and $l$ have a common root (up to a constant, $j_{5}$ is the resultant of $q$ and $l$ ). Without loss of generality, we can suppose that the common factor of $q$ and $l$ is $x$, i.e., $c_{1}=b_{4}=0$ and $c_{0} \neq 0$. Then $j_{7} \sim b_{3}^{2} c_{0}^{4}$, which implies $b_{3}=0$. Then $j_{1} \sim b_{2}^{3}$, which implies $b_{2}=0$. Then $a_{3}$ becomes a factor of $j_{8}$. If $a_{3}=0$, then $j_{3} \sim a_{2}^{2} c_{0}^{2}$, which implies $a_{2}=0$, and then $(l, c, q) \in \mathcal{N}\left(V_{1} \oplus V_{3} \oplus V_{4}\right)$. If $a_{3} \neq 0$, we may take $a_{3}=c_{0}=1$. Now

$$
j_{3} \sim 3 a_{2}^{2}-3 a_{1}-2
$$

and it follows that $a_{1}=a_{2}^{2}-\frac{2}{3}$. Then

$$
j_{6} \sim 27 a_{2}^{3}-54 a_{2}-27 a_{0}-256 b_{1}^{2},
$$

and it follows that $a_{0}=a_{2}^{3}-2 a_{2}-\frac{256}{27} b_{1}^{2}$. Then

$$
j_{4} \sim 36 b_{0}-144 a_{2} b_{1}-949 b_{1}^{3},
$$

and it follows that $b_{0}=4 a_{2} b_{1}+\frac{949}{36} b_{1}^{3}$. Then

$$
j_{2} \sim 27-2048 b_{1}^{4}, \quad j_{8} \sim b_{1}^{5}\left(33205248-4273351745 b_{1}^{4}\right) .
$$

But $j_{2}=j_{8}=0$ has no solution. This settles Case 3 .

By Proposition 1, it follows that these eight invariants form a hsop of the ring of invariants of $V_{1} \oplus V_{3} \oplus V_{4}$.

\subsection{The degrees of the generators}

The Poincaré series of the ring of invariants of $V_{1} \oplus V_{3} \oplus V_{4}$ tells us which is the maximal degree in which we have to look for generators, namely 29. For each $i \leq 29$ we do the following: multiply invariants of smaller degrees to see what part of the vector space of invariants of degree $i$ is known. The Poincaré series tells us how big the dimension of this vector space is, and if the known invariants do not yet span this vector space, one constructs in some way further invariants, until they do span. In the following table $i$ denotes the degree of the generators, and $d_{i}$ the number of generators of degree $i$ needed:

\begin{tabular}{c|cccccccccc}
$i$ & 2 & 3 & 4 & 5 & 6 & 7 & 8 & 9 & 10 & 11 \\
\hline$d_{i}$ & 1 & 2 & 4 & 8 & 10 & 13 & 11 & 10 & 3 & 1
\end{tabular}

For $12 \leq i \leq 29$ no further generators are needed, and it follows that the minimal number of generators is 63 . 


\section{Acknowledgements}

The second author is partially supported by the Swiss National Science Foundation.

\section{References}

[1] Brion M., Invariants de plusieurs formes binaires, Bull. Soc. Math. France 110 (1982), 429-445.

[2] Cayley A., A second memoir upon quantics, Phil. Trans. Royal Soc. London 146 (1856), 101-126.

[3] Derksen H., Kemper G., Computational invariant theory, Encyclopaedia of Mathematical Sciences, Vol. 130, Springer-Verlag, Berlin, 2002.

[4] Gordan P., Beweis, dass jede Covariante und Invariante einer binären Form eine ganze Funktion mit numerischen Coeffizienten einer endlichen Anzahl solcher Formen ist, J. Reine Angew. Math. 69 (1868), 323-354.

[5] Gordan P., Die simultanen Systeme binärer Formen, Math. Ann. 2 (1870), 227-280.

[6] Gundelfinger S., Zur Theorie des simultanen Systems einer cubischen und einer biquadratischen binären Form, Habilitationsschrift, J.B. Metzler, Stuttgart, 1869.

[7] Hammond J., Note on an exceptional case in which the fundamental postulate of professor Sylvester's theory of tamisage fails, Proc. London Math. Soc. 14 (1882), 85-88.

[8] Hilbert D., Ueber die vollen Invariantensysteme, Math. Ann. 42 (1893), 313-373.

[9] Hochster M., Roberts J.L., Rings of invariants of reductive groups acting on regular rings are CohenMacaulay, Adv. Math. 13 (1974), 115-175.

[10] Morley R.K., On the fundamental postulate of tamisage, Amer. J. Math. 34 (1912), 47-68.

[11] Olver P.J., Classical invariant theory, London Mathematical Society Student Texts, Vol. 44, Cambridge University Press, Cambridge, 1999.

[12] Procesi C., Lie groups. An approach through invariants and representations, Universitext, Springer, New York, 2007.

[13] Springer T.A., Invariant theory, Lecture Notes in Mathematics, Vol. 585, Springer-Verlag, Berlin, 1977.

[14] Springer T.A., On the invariant theory of $\mathrm{SU}_{2}$, Indag. Math. 42 (1980), 339-345.

[15] Sylvester J.J., Détermination du nombre exact des covariants irréductibles du système cubo-biquadratique binaire, C. R. Acad. Sci. Paris 87 (1878), 477-481.

[16] Sylvester J.J., Proof of the hitherto undemonstrated fundamental theorem of invariants, Phil. Mag. 5 (1879), 178-188.

[17] Sylvester J.J., Sur le vrai nombre des covariants élémentaires d'un système de deux formes biquadratiques binaires, C. R. Acad. Sci. Paris 84 (1877), 1285-1289.

[18] Sylvester J.J., Sur le vrai nombre des formes irréductibles du système cubo-biquadratique, C. R. Acad. Sci. Paris 87 (1878), 445-448.

[19] Sylvester J.J., Sur les covariants fundamentaux d'un système cubo-quartique binaire, C. R. Acad. Sci. Paris 87 (1878), 287-289.

[20] Sylvester J.J., Franklin F., Tables of the generating functions and groundforms for the binary quantics of the first ten orders, Amer. J. Math. 2 (1879), 223-251. 